\title{
1 CYP2D6 and CYP2A6 biotransform dietary tyrosol into hydroxytyrosol
}

3 Jose Rodríguez-Morató ${ }^{\mathrm{a}, \mathrm{b}, \mathrm{c}}$, Patricia Robledo ${ }^{\mathrm{a}, \mathrm{b}}$, Julie-Anne Tanner ${ }^{\mathrm{d}, \mathrm{e}}$, Anna Boronat ${ }^{\mathrm{a}, \mathrm{b}}$,

4 Clara Pérez-Mañáa,g, C-Y. Oliver Chen ${ }^{\mathrm{h}}$, Rachel F. Tyndale ${ }^{\mathrm{d}, \mathrm{e}, \mathrm{f}}$ and Rafael de la 5 Torre ${ }^{\mathrm{a}, \mathrm{b}, \mathrm{c}, *}$

6

$7 \quad{ }^{a}$ Integrative Pharmacology and Systems Neuroscience Research Group, Neurosciences

8 Research Program. IMIM (Hospital del Mar Medical Research Institute), Dr. Aiguader

9 88, Barcelona 08003, Spain (jrodriguez1@imim.es; probledo@imim.es;

10 aboronat@imim.es; cperez@imim.es; rtorre@imim.es)

$11{ }^{\mathrm{b}}$ Department of Experimental and Health Sciences, Universitat Pompeu Fabra (CEXS-

12 UPF), Dr. Aiguader 80, Barcelona 08003, Spain

$13{ }^{\mathrm{c}}$ Spanish Biomedical Research Centre in Physiopathology of Obesity and Nutrition

14 (CIBEROBN), Instituto Salud Carlos III, 28029 Madrid, Spain

$15{ }^{\mathrm{d}}$ Campbell Family Mental Health Research Institute, Centre for Addiction and Mental

16 Health (CAMH), Toronto, Ontario, Canada (julieanne.tanner@ mail.utoronto.ca)

17 e Department of Pharmacology and Toxicology, Toronto, Ontario, Canada

18 (r.tyndale@utoronto.ca)

$19{ }^{\mathrm{f}}$ Department of Psychiatry, Toronto, Ontario, Canada

$20{ }^{\mathrm{g}}$ Department of Pharmacology, Therapeutics and Toxicology, Autonomous University

21 of Barcelona, Cerdanyola, Spain

$22{ }^{\mathrm{h}}$ Antioxidants Research Laboratory, Jean Mayer USDA Human Nutrition Research

23 Center on Aging, Tufts University, Boston, MA 02111, United States

24 (Oliver.Chen@ tufts.edu) 
25 *Corresponding author: Rafael de la Torre, PharmD, PhD. Integrative Pharmacology

26 and Systems Neuroscience Research Group, IMIM (Hospital del Mar Medical Research

27 Institute), Barcelona, Spain. Tel: +34 933160484; Fax: +34 933160467; E-mail:

28 rtorre@imim.es

29 
31 The dietary phenol tyrosol has been reported to be endogenously transformed into hydroxytyrosol, a potent antioxidant with multiple health benefits. In this work, we evaluated whether tyrosine hydroxylase (TH) and cytochrome P450s (CYPs) catalyzed this process. To assess $\mathrm{TH}$ involvement, Wistar rats were treated with $\alpha$-methyl- $L$ tyrosine and tyrosol. Tyrosol was converted into hydroxytyrosol whilst $\alpha$-methyl- $L$ tyrosine did not inhibit the biotransformation. The role of CYP was assessed in human liver microsomes (HLM) and tyrosol-to-hydroxytyrosol conversion was observed. Screening with selective enzymatic CYP inhibitors identified CYP2A6 as the major isoform involved in this process. Studies with baculosomes further demonstrated that

40 CYP2D6 and CYP3A4 could transform tyrosol into hydroxytyrosol. Experiments using 41 human genotyped livers showed an interindividual variability in hydroxytyrosol 42 formation and supported findings that CYP2D6 and CYP2A6 mediate this reaction. The 43 dietary health benefits of tyrosol-containing foods remain to be evaluated in light of 44 CYP pharmacogenetics.

46 Keywords: Hydroxytyrosol; tyrosol; human liver microsomes; CYP2A6; CYP2D6; 47 metabolism.

\section{Chemical compounds}

49 Hydroxytyrosol (PubChem CID: 82755); Tyrosol (PubChem CID: 10393); Nicotine 50 (PubChem CID: 942), Coumarin (PubChem CID: 323); Dextromethorphan (PubChem 51 CID: 6916184); Tryptamine (PubChem CID: 1150); Methoxsalen (PubChem CID: 52 4114); Tranylcypromine (PubChem CID: 19493). 


\section{Introduction}

Hydroxytyrosol [HT, 2-(3,4-dihydroxyphenyl)ethanol], the main phenolic compound found in olives, virgin olive oil, and red wine, is also a product of dopamine oxidative metabolism (DOPET) (Figure 1A). The health benefits attributed to HT bioactivities include antioxidant, anti-inflammatory, cardioprotective, antitumor, antimicrobial, antidiabetic, and neuroprotective ones (Fernández-Mar, Mateos, GarcíaParrilla, Puertas, \& Cantos-Villar, 2012; Rodríguez-Morató, Xicota, Fitó, Farré, Dierssen, \& de la Torre, 2015).

Based on evidence that the phenolic compounds in olive oil are protective against LDL oxidation (Covas, de la Torre, \& Fitó, 2015), the European Food Safety Authority (EFSA) released a claim regarding the beneficial effects of the daily ingestion of phenolic compound-rich virgin olive oil on cardiovascular disease risk factors. To achieve such benefits a daily dose of $5 \mathrm{mg}$ HT and its derivatives (including tyrosol (Tyr) and oleuropein) in olive oil is required which has opened up the possibility of employing HT and Tyr as nutraceuticals.

The addition of a hydroxyl group at position 3 of the phenol ring provides HT with $o$-diphenol moiety, a key factor for its presenting higher antioxidant activity than Tyr (Carrasco-Pancorbo et al., 2005). Tyr content in red wine has been reported to be more than 5-fold greater than that of HT (Piñeiro, Cantos-Villar, Palma, \& Puertas, 2011), nevertheless, a significant amount of HT has been observed to be endogenously produced. De la Torre, Covas, Pujadas, Fitó, and Farré (2006) described that urinary HT excretion was 1.4-fold higher after the consumption of $250 \mathrm{~mL}$ of red wine compared to $25 \mathrm{~mL}$ of virgin olive oil, despite the HT content being 5-times lower $(0.35 \mathrm{vs} 1.70 \mathrm{mg}$, respectively). Moreover, urinary recovery of HT following red wine administration was greater than the amount of HT present in the wine itself, suggesting an endogenous HT 
production. An ethanol dose-related increase of urinary HT excretion in 24 healthy men

79 has been observed, probably through the induction of endogenous HT production

80 (Pérez-Mañá et al., 2015). Such findings are consistent with previous work (Tank \&

81 Weiner, 1979) in which ethanol up-regulated dopamine oxidative metabolism,

82 generating HT in a dose-dependent manner (Figure 1A).

83 Nevertheless, this ethanol-induced mechanism does not completely explain the

84 amount of HT recovered in urine after wine intake. In a rat study it was reported that

85 Tyr was converted in vivo to HT, and that Tyr urinary excretion was augmented by

86 ethanol (Pérez-Mañá et al., 2015). Despite the fact that Tyr has been identified as a

87 substrate of HT production, the enzyme responsible for this biotransformation remains

88 as yet unknown. Two theoretical possibilities have been considered: the involvement of

89 [i] tyrosine hydroxylase and/or [ii] cytochrome P450 (CYP) isoenzymes (Figure 1B).

and it catalyzes the aromatic hydroxylation that converts $L$-tyrosine to $L$-DOPA

92 (Nagatsu, Levitt, \& Udenfriend, 1964). Taking into account that the difference between

93 Tyr and HT is an aromatic hydroxylation, our first hypothesis was that tyrosine

94 hydroxylase was involved in the conversion of Tyr to HT. Alternatively, we considered

95 CYPs which are responsible for catalyzing the oxidation of a wide variety of xenobiotic chemicals and endogenous substrates.

In addition to identifying the enzymes taking part in Tyr-HT biotransformation,

98 we aimed to evaluate the effects of a combined administration of ethanol and Tyr on the

99 urinary recovery of both Tyr and HT. To this end, the effects of the tyrosine 100 hydroxylase inhibitor $\alpha$-methyl- $L$-tyrosine $(\alpha \mathrm{MT})$ on this biotransformation was assessed in rats; the involvement of CYPs was examined in human liver microsomes 
102 (HLM) and human recombinant proteins (baculosomes); and Tyr hydroxylation was 103 investigated in human genotyped livers.

104 


\section{Material and Methods}

\subsection{Drugs and Chemicals}

HT and HT acetate were supplied by Seprox Biotech (Madrid, Spain). HT-D 3

was purchased from Synfine Research Inc (Ontario, Canada). Tyr, 3-(4-

109 hydroxyphenyl)-1-propanol, homovanillyl alcohol, fluvoxamine maleate, montelukast

110 sodium hydrate, ticlopidine hydrochloride, methoxsalen, tryptamine hydrochloride,

111 omeprazole, ketoconazole, coumarin, NADPH, sodium phosphate monobasic

112 monohydrate, sodium phosphate dibasic anhydrous, paroxetine, fluoxetine, $o$ -

113 phosphoric acid (85\%), nicotine, cotinine, cotinine- $\mathrm{D}_{3}$, coumarin, 7-hydroxycoumarin,

114 4-hydroxycoumarin, dextromethorphan, dextrorphan, 2-benzoxazolinone, and dimethyl

115 sulfoxide were purchased from Sigma-Aldrich Corporation (St. Louis, MO, USA).

116 Sulfaphenazole and tranylcypromine hydrochloride were purchased from Cayman

117 Chemical (Ann Arbor, MI, USA). HT glucuronide conjugates were synthesized

118 according to a previously described method (Khymenets, Joglar, Clapés, Parella, Covas,

$119 \&$ de la Torre, 2006). HT-D 4 was custom synthesized by Toronto Research Chemicals

120 Inc. (Toronto, Ontario, Canada). HT-1-O-sulfate was custom synthesized by Industrial

121 Research Limited (Lower Hutt, New Zealand). Methanol and acetonitrile (HPLC 122 gradient grade), ammonium hydroxide, and acetic acid were purchased from Merck

123 (Darmstadt, Germany). Ultrapure water was obtained using a Milli-Q purification 124 system (Millipore Ibérica, Barcelona, Spain). Standard stock solutions of Tyr, HT, and

125 their metabolites, as well as their corresponding internal standards (IS), were prepared 126 using $10 \mathrm{mg} / \mathrm{mL}$ methanol. Working solutions were obtained by further diluting the 127 stock solutions and were then stored in amber vials at $-20^{\circ} \mathrm{C}$. 
131 Life Technologies Europe. According to the manufacturer, total protein and P450 132 content were $20.0 \mathrm{mg} / \mathrm{mL}$ and $0.239 \mathrm{nmol} / \mathrm{mg}$, respectively. Microsomes produced from 133 baculovirus-infected insect cells expressing human CYP2A6, CYP3A4, CYP2B6, 134 CYP2C9, and CYP2D6 (Baculosomes ${ }^{\circledR}$ ) were also purchased from Life Technologies 135 Europe with the corresponding protein concentrations and CYP contents.

136 The characteristics and sources of the 15 human livers used in this study have 137 been previously described (Messina, Tyndale, \& Sellers, 1997). Human hepatic 138 microsomes were prepared and stored in $1.15 \% \mathrm{KCl}$ at $-80^{\circ} \mathrm{C}$ according to previously 139 established techniques (Tyndale, Inaba, \& Kalow, 1989). Human liver tissues were 140 derived from the K- and M-series liver banks provided by Dr. T. Inaba (University of 141 Toronto, Toronto, ON, Canada) and Dr. U. Meyer (Biocentre in Basel, Switzerland), 142 respectively, as previously published (Al Koudsi, Hoffmann, Assadzadeh, \& Tyndale, 143 2010; Messina, Tyndale, \& Sellers, 1997). Cytosolic fractions were collected during 144 microsomal membrane preparation and used as a source of aldehyde oxidase for all in 145 vitro nicotine metabolism assays. Total protein was quantified with a Bio-Rad protein 146 assay kit based on the Bradford dye-binding method (Bio-Rad Laboratories Ltd.).

147 DNA was extracted from liver tissues for CYP2A6 and CYP2D6 genotyping with 148 phenol/chloroform extraction (Invitrogen, Canada) and ethanol precipitation. DNA was 149 genotyped for the CYP2A6 alleles $* 2, * 4, * 5, * 6, * 9, * 12, * 17, * 20, * 21, * 23, * 24, * 25$, $150 * 26, * 27, * 28, * 35, * 1 X 2$, and the $C Y P 2 D 6$ alleles $* 3, * 4, * 10$, as previously described 151 (Tyndale, Droll, \& Sellers, 1997; Wassenaar, Zhou, \& Tyndale, 2015). Liver donors 152 were then grouped as normal or reduced CYP2A6 and CYP2D6 metabolizers based on 153 predicted activity according to genotype. The CYP2A6 and CYP2D6 normal groups 154 included donors of genotypes $C Y P 2 A 6^{*} 1 / * 1, * 1 / 1 X 2$ and $C Y P 2 D 6^{*} 1 / * 1$ only for each 
gene. CYP2A6 protein levels in these human liver tissues were semi-quantified with

156 Western blotting, as previously described (Al Koudsi, Hoffmann, Assadzadeh, \&

157 Tyndale, 2010).

\subsection{Animal studies}

Seventy-two male Wistar rats (Charles River) weighing 140-200 g were used in the experiments. They were housed two per cage (temperature $22 \pm 2^{\circ} \mathrm{C} /$ humidity $55 \pm$ $15 \%$ ) in a controlled room with a 12-h light/dark cycle (lights on at 08:00 h). The experiments were performed during the period of light. Food and water were given $a d$ libitum in the home cages, but only water was available in the metabolic cages (Harvard

165 Apparatus, $48 \mathrm{~cm} \times 28 \mathrm{~cm} \times 36 \mathrm{~cm}$ ) during the four hours of the experiment. Each cage 166 was provided with a support grid for the animals, separate urine and feces collection 167 funnels, and a drinking tube. On the day prior to the experimental session, the rats were habituated to the metabolic cages for two hours. They were assigned to twelve treatments $(\mathrm{N}=6$ /group) (Supplementary Table 1$)$. The rats were first injected with 170 either $\alpha$ MT $(50 \mathrm{mg} / \mathrm{kg})$ or saline, and thirty min later received either $0.5 \mathrm{~g} / \mathrm{kg}$ ethanol 171 (30\% ethanol in saline, v/v) or vehicle (saline). One hour later, they were injected with 10 or $20 \mathrm{mg} / \mathrm{kg}$ of Tyr (prepared in saline) or vehicle. All treatments were administered intraperitoneally. Following the last administration, the rats were immediately placed in

174 the cages for $4 \mathrm{~h}$. and urine produced during this period collected. On terminating the 175 experiment, the rats were euthanized under isoflurane anesthesia. Urine samples were 176 weighed and preserved with $6 \mathrm{~N} \mathrm{HCl}(20 \mu \mathrm{L} / \mathrm{mL}$ urine $)$, and stored at $-20^{\circ} \mathrm{C}$ until 177 analysis. HT, Tyr, and their corresponding metabolites were quantified using a LC178 MS/MS method as previously described (Khymenets et al., 2010; Kotronoulas et al., 179 2013; Pérez-Mañá et al., 2015). Animal procedures were approved by the local ethical 
180 committee (CEEA-PRBB; ref. PRM-13-1525) and performed in accordance with the 181 guidelines of the European Communities Directive 86/609/EEC regulating animal 182 research.

\subsection{Experiments in Human Liver Microsomes (HLM)}

\subsubsection{Tyrosol metabolism to hydroxytyrosol}

The evaluation of HT formation was carried out at $37^{\circ} \mathrm{C}$ in a shaking bath for 0 to $60 \mathrm{~min}$. The final incubation mixtures $(250 \mu \mathrm{L})$ contained Tyr $(10-100 \mu \mathrm{M})$, HLM (0.5 mg protein/mL), and NADPH $(1 \mathrm{mM})$ in $100 \mathrm{mM}$ sodium phosphate buffer $(\mathrm{pH}$ 7.4). The reactions were initiated by the addition of the NADPH solution after a 3-min

190 pre-incubation, and stopped at each corresponding time by the addition of $250 \mu \mathrm{L}$ ice-

191 cold methanol. A negative control in the absence of NADPH was employed to verify the NAPDH-dependent reaction. To each reaction mixture, $20 \mu \mathrm{L}$ IS solution (10 $\mu \mathrm{g} / \mathrm{mL}$ each of HT- $\mathrm{D}_{3}$ and 3-(4-hydroxyphenyl)-1-propanol) was added. The resulting

194 mixture was centrifuged $\left(13,000 \mathrm{rpm}, 5 \mathrm{~min}, 4^{\circ} \mathrm{C}\right)$. The supernatant was transferred to 195 an amber screw-top glass tube, diluted with $4.25 \mathrm{~mL}$ of Milli-Q water, acidified to $\mathrm{pH} 2$ 196 with $250 \mu \mathrm{L} 4 \% \mathrm{H}_{3} \mathrm{PO}_{4}$, and subjected to a solid-phase extraction (SPE) procedure 197 (Oasis HLB $^{\odot} 3 c c, 60-m g$ cartridges; Waters Corporation, Dublin, Ireland). The SPE 198 cartridges were preconditioned sequentially with $2 \mathrm{~mL}$ methanol and $2 \mathrm{~mL}$ water. After 199 sample loading, the cartridges were washed with $2 \mathrm{~mL}$ water. The compounds of 200 interest were then eluted with $3 \mathrm{~mL}$ methanol. After methanol removal $\left(25^{\circ} \mathrm{C}, 10-15\right.$ 201 psi), the dry residues were reconstituted in a $100 \mu \mathrm{L}$ mixture of mobile phases $202(90 \% \mathrm{~A} / 10 \% \mathrm{~B}, \mathrm{v} / \mathrm{v})$, centrifuged at 10,000 rpm for $3 \mathrm{~min}$, transferred to HPLC vials, and analyzed by LC-MS/MS. This procedure was employed for both the mix of HLM and

204 the individual genotyped microsomes. 

1200 series HPLC system (Agilent technologies) coupled to a triple quadrupole (6410

207 Triple Quad LC/MS; Agilent) mass spectrometer with an electrospray interface.

208 Chromatographic separation of HT and Tyr was carried out on an Acquity UPLC ${ }^{\circledR}$ BEH

$209 \mathrm{C}_{18}$ column (100 $\mathrm{mm} \times 3.0 \mathrm{~mm}$ i.d., $1.7 \mu \mathrm{m}$ particle size) (Waters Corporation) at $40^{\circ} \mathrm{C}$ 210 in an isocratic mode using $75 \%$ mobile phase A $(0.01 \%$ ammonium acetate, $\mathrm{pH} 5)$ and $21125 \%$ mobile phase B (100\% methanol). Injection volume was $10 \mu \mathrm{L}$. Tyr, HT, and the

212 IS were eluted with the flow rate at $0.25 \mathrm{~mL} / \mathrm{min}$ in $5 \mathrm{~min}$ and monitored in negative 213 ionization using the multiple reaction mode. HT was quantified employing an isotope 214 dilution method by comparing its peak area ratio with HT- $\mathrm{D}_{3}$.

\subsubsection{Nicotine metabolism to cotinine}

The rates of nicotine, coumarin, and dextromethorphan metabolism were assessed in vitro in the HLMs from the 15 donors. The velocity of nicotine metabolism to cotinine was determined by incubating microsomal protein $(0.5 \mathrm{mg} / \mathrm{mL})$ with nicotine $(30 \mu \mathrm{M})$, Tris- $\mathrm{HCl}$ buffer $(\mathrm{pH} 7.4,50 \mathrm{mM})$, NADPH $(1 \mathrm{mM})$, and $10 \mu \mathrm{L}$ of human liver cytosol (as the aldehyde oxidase source) in the final volume of $100 \mu \mathrm{L}$. The reactions were carried out for $20 \mathrm{~min}$ at $37{ }^{\circ} \mathrm{C}$ and stopped with the addition of $20 \mu \mathrm{L}$ of $20 \% \mathrm{Na}_{2} \mathrm{CO}_{3}$. The IS, cotinine- $\mathrm{D}_{3}$, was added. Samples were extracted and analyzed by LC-MS/MS as previously described (Tanner et al., 2015).

\subsubsection{Coumarin metabolism to 7-hydroxycoumarin}

Coumarin metabolism to 7-hydroxycoumarin was evaluated by incubating microsomal protein $(0.05 \mathrm{mg} / \mathrm{mL})$ with coumarin $(2 \mu \mathrm{M})$, Tris-HCl buffer $(\mathrm{pH} 7.4,50$ $\mathrm{mM})$, and NADPH $(1 \mathrm{mM})$ in the final volume of $200 \mu \mathrm{L}$. The rate of 7 hydroxycoumarin formation was linear from 2-15 min; for fast CYP2A6 livers, according to the rate of cotinine formation from nicotine (highest tertile), shorter 
230 incubation times were used to avoid substrate depletion. The reactions were carried out 231 at $37^{\circ} \mathrm{C}$ and stopped with the addition of $40 \mu \mathrm{L}$ trichloroacetic acid $(20 \% \mathrm{w} / \mathrm{v})$.

232 Following the addition of the IS, 4-hydroxycoumarin, the resulting reaction mixtures 233 were extracted and analyzed by HPLC as previously described (Li, Li, \& Sellers, 1997), 234 with minor modifications. Briefly, after the addition of $3 \mathrm{~mL}$ ethyl acetate, the mixtures were vortexed for 10 seconds, mechanically shaken for $10 \mathrm{~min}$, and centrifuged at 3,000 rpm for $10 \mathrm{~min}$. The upper ethyl acetate layer was then transferred to a $10-\mathrm{mL}$ tube and evaporated to dryness at $37^{\circ} \mathrm{C}$ under nitrogen stream. The dry residue was re-dissolved with $110 \mu \mathrm{L}$ mobile phase, and $100 \mu \mathrm{L}$ of the solution was injected into the HPLC system (HP 1200 Separation Module). Coumarin and 7-hydroxycoumarin were separated on the ZORBAX SB C18 Column $(5 \mu \mathrm{m} .250 \times 4.6 \mathrm{~mm}$; Agilent

241 Technologies, Mississauga, ON) using mobile phase of acetonitrile, water and acetic acid (25:75:0.1, v/v) at $1 \mathrm{~mL} / \mathrm{min}$ flow rate. The retention time of 7-hydroxycoumarin, 4-hydroxycoumarin, and coumarin was 7.9, 11.8, and $17.7 \mathrm{~min}$, respectively.

\subsubsection{Dextromethorphan metabolism to dextrorphan}

The metabolism of dextromethorphan to dextrorphan was measured by incubating microsomal protein $(0.25 \mathrm{mg} / \mathrm{mL})$ with dextromethorphan $(5 \mu \mathrm{M}), 100 \mathrm{mM}$ potassium phosphate buffer $(\mathrm{pH} 7.4)$, and NADPH $(1 \mathrm{mM})$ in the final volume of 250 $\mu \mathrm{L}$. The reactions were carried out for $15 \mathrm{~min}$ at $37^{\circ} \mathrm{C}$ and stopped with the addition of $250 \mu \mathrm{L}$ hexane-butanol (95:5 v/v). The IS, 2-benzoxazolinone, was added. The resulting mixtures were extracted and analyzed by HPLC as previously described (Flores-Pérez, Flores-Pérez, Juárez-Olguín, Lares-Asseff, \& Sosa-Macías, 2004; Hendrickson, Gurley,

252 \& Wessinger, 2003), with minor modifications. Specifically, $5 \mathrm{~mL}$ hexane-butanol 253 (95:5 v/v) was first added, and the same procedure used for coumarin (see subheading 
with a ZORBAX Bonus-RP column $(5 \mu \mathrm{m}, 250 \times 4.6 \mathrm{~mm}$; Agilent Technologies,

256 Mississauga, ON) under a gradient elution condition using solvent A (methanol and

$2570.05 \mathrm{M}$ phosphate buffer, 45:55 v/v, $\mathrm{pH} 5.8$ ) and solvent $\mathrm{B}$ (water) at room temperature.

258 The linear gradient from $100 \%$ to $70 \%$ solvent A was applied from 0 to 14 min at a flow 259 rate of $0.8 \mathrm{~mL} / \mathrm{min}$. From 14 to $27 \mathrm{~min}$, the mobile phase was kept constant at $100 \%$

260 solvent $\mathrm{A}$ and $1.2 \mathrm{~mL} / \mathrm{min}$ flow rate. The eluents were monitored by a fluorescence 261 detector set at an excitation/emission wavelength of 230/330 $\mathrm{nm}$. Retention time was 26210.52 , 13.26, and 22.63 min for dextrorphan, 2-benzoxazolinone, and dextromethorphan, 263 respectively.

\subsection{Enzyme Kinetics Experiments}

Tyr, at concentrations ranging from 0.1 to $4000 \mu \mathrm{M}$, was incubated with HLM and the procedure described in the subheading 2.4.1. was followed. All kinetic analyses were performed in duplicate. The kinetic parameters $\left(\mathrm{K}_{\mathrm{m}}\right.$ and $\left.\mathrm{V}_{\max }\right)$ were estimated using the Michaelis-Menten equation generated by GraphPad Prism (GraphPad Software, CA, USA, version 5.03 for Windows). min, respectively. These time-points were chosen based on preliminary experiments evaluating enzymatic reaction linearity (Supplementary Figure 1). The curves describing the reaction kinetics were analyzed using non-linear regression analysis (GraphPad Software 5.03, CA, USA).

\subsection{Chemical Inhibition Analyses}

The effects of specific CYP inhibitors on tyrosol hydroxylase activity in HLM 
$280(0.5 \mathrm{mg} / \mathrm{mL})$ and NADPH $(1 \mathrm{mM})$ in the absence (positive control) and presence of 281 selective chemical inhibitors of CYP isoforms. All the chemical inhibition studies were 282 performed in duplicate. The following CYP inhibitors were used: fluvoxamine for 283 CYP1A2, ticlopidine for CYP2B6, montelukast for CYP2C8, sulfaphenazole for 284 CYP2C9, omeprazole for CYP2C19, quinidine for CYP2D6, and ketoconazole for 285 CYP3A4. In the case of CYP2A6, the substrate coumarin was employed (See 286 Supplementary Figure 2 for the chemical structures). The specific inhibitors were 287 chosen following the European Medicine Agency guidelines on the Investigation of 288 Drug Interactions (EMA, 2012). The following additional inhibitors were also used in 289 confirmatory experiments with tryptamine, tranylcypromine, and methoxsalen as 290 CYP2A6 inhibitors, and paroxetine and fluoxetine as CYP2D6 inhibitors. The range of 291 inhibitor concentrations was 1-100 $\mu \mathrm{M}$. Initially, stock solutions of CYP inhibitors were prepared in DMSO. The final DMSO concentration in the reactions was $1.0 \%$. Due to a remarked DMSO-induced inhibitory effect on the reaction, working inhibitor solutions were prepared in methanol. In order to avoid any interference from organic solvent, the

295 inhibitors were prepared in methanol to facilitate solvent removal for the assay. 296 Methanol was evaporated in a vacuum concentrator $\left(\operatorname{SpeedVac}{ }^{\circledR}\right)$. The reaction mixture excluding NADPH was added to tubes containing inhibitors. The reactions were started by the addition of NADPH and were stopped at $60 \mathrm{~min}$ by the addition of $250 \mu \mathrm{L}$ icecold methanol.

Additional inhibitory experiments were performed to assess the effect of 301 mechanism-based inhibitors (methoxsalen for CYP2A6, paroxetine for CYP2D6) on 302 HT formation. In these cases, the inhibitor was pre-incubated with microsomes and 303 NADPH for 30 min prior to the addition of the substrate. The reaction mixture was then 304 incubated at $37^{\circ} \mathrm{C}$ for another $30 \mathrm{~min}$, and treated as previously described. 


\subsection{HT formation in cDNA-Expressing CYP microsomes}

Incubations were carried out at $37^{\circ} \mathrm{C}$ in a shaking bath for 0 to $60 \mathrm{~min}$. The final incubation mixtures $(250 \mu \mathrm{L})$ contained Tyr $(40-400 \mu \mathrm{M})$, the corresponding cDNAexpressing CYP microsomes (40-80 pmol CYP/mL), and NADPH (1 mM) in $100 \mathrm{mM}$

310 sodium phosphate buffer $(\mathrm{pH}$ 7.4). The reactions were started by the addition of

311 NADPH solution after a 3-min pre-incubation and stopped at selected times (0-60 min)

312 by the addition of $250 \mu \mathrm{L}$ ice-cold methanol. A negative control in the absence of

313 NADPH was employed to verify the NAPDH-dependent reaction. Prior to analysis, the

314 samples were processed as described for HLM samples.

\subsection{Statistical Analyses}

Data analyses of animal and chemical inhibition studies were performed using

a three-way ANOVA model (to evaluate effects of Tyr, ethanol, and $\alpha \mathrm{MT}$ on HT urinary excretion) as well as a two-way ANOVA (to assess the effects of Tyr and ethanol on Tyr urinary excretion). Post hoc multiple comparisons were performed for statistically significant results in the global analyses with the LSD test. The correlations between (1) HT formation from Tyr, and 7-hydroxycoumarin formation from coumarin, and (2) HT formation from Tyr, and dextrorphan formation from dextromethorphan were determined by Spearman's coefficient. Associations between HT production and CYP2A6 genotype and CYP2D6 genotype were analysed with the Mann-Whitney test. Statistical analyses were performed using SPSS Statistics for Windows (Version 21.0; SPSS Inc. Chicago, IL, USA). The level of statistical significance was defined as $P$ $<0.05$. The results in rats are expressed as mean \pm S.E.M., and the in vitro findings as mean \pm S.D. 
331 regression analysis, and 3D graphics were performed with MATLAB software version

332 7.0. Experimental data were fitted to a quadratic equation including two linear terms 333 and a quadratic one in order to obtain the optimum correlation. 


\section{Results}

336

337

338

339

340

341

\subsection{Animal experiments}

A dose-dependent increase in total Tyr urinary recovery was found following Tyr administration (Figure 2A). A two-way ANOVA revealed a main effect of Tyr $\left(F_{(2,28)}=92.2, p<0.001\right)$ although no significant effect of ethanol or interaction between factors were observed, indicating that ethanol does not modify the excretion of Tyr (Figure 2A).

A three-way ANOVA evaluating the action of Tyr, ethanol, and aMT on total HT urinary recovery showed a main effect of Tyr $\left(\mathrm{F}_{(2,42)}=23.7, \mathrm{p}<0.001\right)$ and ethanol $\left(\mathrm{F}_{(1,42)}=11.1, \mathrm{p}<0.001\right)$, but none of $\alpha \mathrm{MT}$. No interactions amongst factors were observed. However, the results in Figure $2 \mathrm{~B}$ demonstrate that Tyr increased total urinary HT recovery in a dose-dependent fashion, while $\alpha \mathrm{MT}$ did not significantly inhibit this effect at the dose studied. Ethanol slightly inhibited the conversion of Tyr to HT without reaching statistical significance. The combination of $\alpha \mathrm{MT}$ and ethanol also decreased HT formation although this effect was mainly due to the inhibitory action of ethanol (Figure 2B).

\subsection{Tyrosol hydroxylase activity in HLM}

HT formation from Tyr took place in human hepatic microsomes in an NADPHdependent manner and increased in a linear fashion $\left(r^{2}>0.98\right)$ from 0 to 60 min reaction time. This hydroxylation activity occurred in a substrate concentration-dependent manner (Figure 3A).

\subsection{Kinetic analyses of Tyrosol ortho-hydroxylation in HLM}


Figure 3 shows the Michaelis-Menten, Lineweaver-Burk, and Eadie-Hofstee

360 plots for the HLM-mediated HT formation from Tyr. The values represent the mean of

361 two separate experiments. The apparent $\mathrm{K}_{\mathrm{m}}$ value was $709 \pm 49 \mu \mathrm{M}$ and the $\mathrm{V}_{\max }$ value was $1294 \pm 31 \mathrm{pmol} / \mathrm{min} / \mathrm{mg}$.

\subsection{Chemical inhibition studies with specific CYP inhibitors}

The effect of 8 CYP inhibitors on HT formation from Tyr was evaluated. Firstly,

a primary screening using 8 specific inhibitors at $100 \mu \mathrm{M}$ was performed. Figure 4 shows the mean activities (from duplicate determinations) in the presence of these inhibitors. Coumarin reduced HT formation from Tyr (100 $\mu \mathrm{M})$ by $60 \%$. CYP2D6, CYP3A4, CYP2B6, and CYP2C9 selective inhibitors slightly diminished HT formation $(>10 \%$ but $<20 \%)$ (Figure $4 \mathrm{~A})$.

Additional incubations using nicotine as a CYP2A6 substrate and tryptamine, methoxsalen, and tranylcypromine as CYP2A6 inhibitors (Zhang, Kilicarslan, Tyndale, \& Sellers, 2001) were performed. Although nicotine was not found to decrease HT

374 formation, three CYP2A6 inhibitors reduced the biotransformation to a larger degree 375 than coumarin $(74 \%, 77 \%$, and $85 \%$, respectively), with tranylcypromine being the strongest (Figure 4B). It is worth noting that the rate of coumarin metabolism by CYP2A6 is relatively fast compared to the other substrates/inhibitors tested.

379 (tranylcypromine, methoxsalen) were pre-incubated with NADPH and microsomes for

$38030 \mathrm{~min}$ and, after the addition of Tyr, the reactions took place for 30 additional min.

381 Pre-incubation with the CYP2D6 inhibitors did not lead to a $>20 \%$ inhibition, whereas 382 the CYP2A6 inhibitors surpassed 90\%. It is of interest that the combination of two 383 mechanism-based inhibitors, paroxetine (CYP2D6) and methoxsalen (CYP2A6), 
384 inhibited HT formation by $97 \%$ (Figure 4C). No inhibitory effect was observed when a

385 deuterated analog of $\mathrm{HT}\left(\mathrm{HT}-\mathrm{D}_{4}\right)$ was added at 1,10 , and $100 \mu \mathrm{M}$ to the reaction

386 mixture, indicating there was no product-initiated inhibition of the reaction.

387

\subsection{Experiments using human recombinant CYP enzymes (Baculosomes)}

HT formation from Tyr was evaluated in baculosomes (microsomes from

390 baculovirus-infected insect cells expressing human isoforms). CYP2A6 capacity for hydroxylating tyrosol was confirmed, and HT was found to be produced in a linear fashion $\left(r^{2}>0.99\right)$ with the amount of administered protein and Tyr (Supplementary

\section{Figure 3).}

In order to evaluate whether other CYP isoforms could contribute to HT

395 production, human recombinant CYP3A4, CYP2B6, CYP2C9, and CYP2D6 (as well as CYP2A6) were tested. These isoforms were chosen based on the results of the chemical inhibition assays (Figure 4A) in which the treatment with specific inhibitors of these isoenzymes decreased by HT formation by at least 10\%. All the five human recombinant CYP isoforms evaluated were capable of transforming Tyr into HT with

400 CYP2D6, CYP3A4, and CYP2A6 being more reactive than CYP2B6 and CYP2C9

401 (Supplementary Figure 4). In all the cases, HT formation was NADPH-dependent. contributors) were evaluated individually at 6 time points $(0,5,15,30,45$, and $60 \mathrm{~min})$.

404 Differing kinetics were noted for each enzyme. CYP2A6-mediated HT formation 405 occurred in a linear time-dependent manner from 0 to $60 \mathrm{~min}$. The linearity in HT 406 formation mediated by CYP3A4 and CYP2D6 was observed only in the first 15 min, 407 and there was a time-dependent reduction in HT content from 15 to $60 \mathrm{~min}$ 408 (Supplementary Figure 1). 
410 evaluated in CYP2A6 and CYP2D6 baculosomes with the aim of comparing their

411 kinetic parameters with those obtained using HLM. HT formation kinetics in

412 baculosomes did not follow a typical Michaelis-Menten hyperbolic pattern. In the case

413 of CYP2A6, a biphasic kinetic profile was observed (Supplementary Figure 5A). This

414 enzymatic behavior has been previously described (Hutzler \& Tracy, 2002) and is

415 characterized as a non-asymptotic profile that becomes linear with increasing substrate

416 concentration. With respect to CYP2D6, the kinetics followed a sigmoidal

417 autoactivation profile (Supplementary Figure 5B).

418

419 3.6. Correlation of Tyr Hydroxylation by Individual HLM

HT formation from Tyr was evaluated in 15 individual genotyped livers, which exhibited a wide range of CYP2D6 and CYP2A6 activities. Table 1 shows the individual genotypes for CYP2D6 and CYP2A6 and the predicted activities of both isoforms according to genotype.

The correlation between HT formation and CYP2A6 and CYP2D6 activities 425 (determined using the metabolism velocity of coumarin to 7-hydroxycoumarin, and 426 dextromethorphan to dextrorphan, respectively) was non-significant (Figure 5A and B).

427 The impact of genotype on the association between HT formation and CYP2A6 and CYP2D6 genotypes was non-significant (Figure 5C and D).

In order to evaluate the concomitant involvement of both isoforms on HT 430 formation, we performed a multivariate regression analysis. A three-dimensional scatter 431 plot for the sample-to-sample variation in Tyr hydroxylation of the 15 individual HLMs, 432 and their correlations with the individual CYP2D6 and CYP2A6 activities, is depicted 
433 in Figure 5E. The equation of the adjusted surface that describes the velocity of Tyr

434 hydroxylation is:

Tyr hydroxylation $=0.01263+0.52778 \cdot X_{1}+0.044483 \cdot X_{2}-0.57453 \cdot X_{1} \cdot X_{2}$

Where $X_{1}$ is dextromethorphan velocity and $X_{2}$ is coumarin velocity. The corresponding correlation coefficient $\left(\mathrm{r}^{2}\right)$ is $0.50(\mathrm{~F}=3.69 ; P=0.047)$. It should be noted that the regression coefficient values were comparable when CYP2A6 velocity toward either coumarin or nicotine was included in the analysis.

\section{Discussion}

Recent studies have demonstrated that HT is endogenously formed from Tyr in both rats and humans (Pérez-Mañá et al., 2015). However, the enzymes that mediated 444 the conversion remained to be characterized. To the best of our knowledge, we demonstrate for the first time that CYPs convert Tyr into HT, and that this reaction is primarily mediated by two polymorphic CYP isoenzymes, CYP2D6 and CYP2A6. Furthermore, we observed that tyrosine hydroxylase was not involved in the conversion.

We hypothesized that the conversion of Tyr to HT was mediated by either

449 tyrosine hydroxylase or CYPs. In order to examine the possible role of tyrosine 450 hydroxylase in the formation of HT from Tyr, rates were pretreated with $\alpha \mathrm{MT}$, a 451 tyrosine hydroxylase inhibitor, before Tyr administration. The unchanged urinary HT 452 excretion provided strong evidence that tyrosine hydroxylase was not involved in the 453 conversion of Tyr to HT. In the same rat study, it was confirmed that urinary HT 454 excretion occurred in parallel to the administered Tyr doses, while $0.5 \mathrm{~g} / \mathrm{kg}$ ethanol 455 slightly inhibited this effect. It is noteworthy that ethanol plays a dual role: it alters 456 dopamine oxidative metabolism generating small amounts of HT, whilst appearing to 457 inhibit the conversion of Tyr into HT. 
459 formation, we next tested whether CYPs were capable of mediating Tyr hydroxylation.

460 In vitro HLM experiments confirmed that at least one CYP isoform took part in the

461 conversion of Tyr to HT via hydroxylation with a typical Michaelis-Menten kinetic

462 profile. Such a combination of high $\mathrm{K}_{\mathrm{m}}$ and $\mathrm{V}_{\max }$ values indicates that, despite

463 presenting a low specificity, Tyr-3-hydroxylation occurs at a high velocity.

464 Once Tyr hydroxylation had been demonstrated to occur in human microsomes,

465 it was necessary to identify the specific CYP isoenzyme(s) that catalyze(s) the reaction.

466 Results from the HLM experiments using selective inhibitors suggested that CYP2A6

467 was the main isoform responsible for the conversion of Tyr to HT, although CYP2D6

468 and CYP3A4 appeared to be involved to a lesser extent. The use of baculosomes

469 (recombinant baculovirus containing cDNA) confirmed the capacity of CYP2A6, 470 CYP2D6, and CYP3A4 to biotransform Tyr to HT.

471 The involvement of CYP3A4 baculosomes in the conversion of Tyr to HT is not 472 surprising as it is the most abundant isoform in the liver, and it metabolizes the greatest 473 number of drugs and other xenobiotics (Pelkonen, Turpeinen, Hakkola, Honkakoski, 474 Hukkanen, \& Raunio, 2008). Nevertheless, according to inhibition experiments with 475 ketoconazole in pooled human liver microsomes, is should be noted that CYP3A4 is not 476 a strong contributor to in vivo HT formation. The role of CYP2A6 and CYP2D6 in the 477 conversion of Tyr to HT appears to be of greater relevance. Findings from HLM 478 experiments using methoxsalen and paroxetine, two mechanism-based inhibitors of 479 CYP2A6 and CYP2D6, respectively, suggest that both isoforms react toward Tyr in a 480 cooperative manner with CYP2A6 as the major contributor and CYP2D6 the minor one. 481 The kinetics of HT formation mediated by CYP2A6 and CYP2D6 was evaluated in 482 baculosomes. Contrary to what occurred in microsomes, HT formation in baculosomes 
483 did not follow the typical hyperbolic Michaelis-Menten kinetic profile. CYP2A6

484 presented a biphasic kinetic profile without saturation, even at high concentrations,

485 whilst CYP2D6 had a sigmoidal autoactivation one. Such atypical kinetic profiles have

486 been previously described in vitro, especially for CYP3A4 (Hutzler \& Tracy, 2002),

487 suggesting that they might result from the simultaneous binding of multiple ligands to a

488 single active site (Atkins, 2005). In our study, the lack of Michaelis-Menten kinetic

489 profiles in baculosomes hindered the comparison of the individual contributions of

490 CYP2A6 and CYP2D6 to HT formation, although at equimolar concentration CYP2D6

491 presented a higher velocity of HT formation than CYP2A6. The discrepancy between

492 the kinetic profile observed in microsomes and baculosomes may be due to the fact that

493 recombinant CYP enzymes are usually expressed with much higher levels of NADPH

494 and CYP reductase than those found in HLMs (Rodrigues, 2008). Moreover, Tyr

495 hydroxylation in a mix of 50 HLMs (presenting a more physiological condition than

496 baculosomes) fully coincided with a typical Michaelis-Menten kinetic profile. Taking

497 into account the previous observations, we suggest that in vivo Tyr hydroxylation

498 follows a Michaelis-Menten kinetic profile.

499 There is high interindividual variability in CYP2A6 and CYP2D6 activities due 500 to genetic polymorphisms (Yokoi \& Kamataki, 1998). CYP2D6, which catalyzes more 501 than $25 \%$ of commercial drugs, has a gene locus with more than 80 allelic variants, 502 resulting in considerable differences associated with increased or reduced enzymatic 503 activity and clinical consequences (Teh \& Bertilsson, 2012). In a similar manner, the 504 CYP2A6 gene is polymorphic, resulting in high interindividual (Rautio, Kraul, Kojo, 505 Salmela, \& Pelkonen, 1992) and interethnic divergence in enzyme activity (Piliguian et 506 al., 2014; Raunio, Rautio, Gullsten, \& Pelkonen, 2001). Indeed, 20\% of Asians are 507 CYP2A6 poor metabolizers, whereas this prevalence is lower in Caucasians $(<1 \%)$ 
508 (Raunio, Rautio, Gullsten, \& Pelkonen, 2001). Regarding the relative abundance of

509 protein in the liver, CYP2D6 accounts for less than 5\% whilst CYP2A6 represents

510 approximately $10 \%$ of total hepatic CYP protein (Pelkonen, Turpeinen, Hakkola,

511 Honkakoski, Hukkanen, \& Raunio, 2008).

512 The specificity of each CYP isoform toward its substrates is another issue that

513 must be taken into account. CYP2D6 and CYP3A4 are responsible for the metabolism

514 of hundreds of therapeutic drugs (Pelkonen, Turpeinen, Hakkola, Honkakoski,

515 Hukkanen, \& Raunio, 2008) whilst relatively few are converted by CYP2A6, the

516 enzyme primarily responsible for nicotine metabolism (Messina, Tyndale, \& Sellers, 517 1997).

518 In this study, CYP2A6 and CYP2D6 from the 15 selected livers were classified

519 according to genotype-predicted activities, and their capacity for nicotine-to-cotinine 520 conversion and dextromethorphan-to-dextrorphan conversion, respectively. As expected,

521 a wide interindividual Tyr-to-HT conversion variability was found. It was also observed

522 that the correlations between HT formation and coumarin (a CYP2A6 substrate) and

523 dextromethorphan (a CYP2D6 substrate) velocity were weak (Spearman $\mathrm{r}=0.28$ in

524 both cases) although there were modest associations between HT formation and 525 CYP2A6/CYP2D6 activities. These results, and the previous findings with respect to 526 baculosomes (reporting the involvement of CYP2D6 and CYP2A6) and inhibition 527 studies with microsomes (demonstrating CYP2A6 function), indicate that both CYP2D6 528 and CYP2A6 are actively involved in HT formation from Tyr. An implication that is 529 further upheld by the larger regression coefficient generated in the multivariate 530 regression analysis with CYP2D6 and CYP2A6 velocities as two independent variables.

$531 \quad$ Our study has strengths and limitations. One strength is the combination of in 532 vivo (animal models) and in vitro (microsomes and baculosomes) techniques employed, 
533 as well as the use of human livers. A limitation is the relatively low number of human

534 livers used due to the difficulty in obtaining these samples.

535

536

\section{Conclusions}

537 In conclusion, our study demonstrates for the first time that HT (a potent 538 bioactive molecule with multiple health benefits) is formed from dietary Tyr in HLMs

539 via CYP2A6 and CYP2D6, both working in a cooperative manner. The involvement of 540 two CYP isoenzymes in the production of HT is relevant since to date only a few 541 known substrates of CYP2A6 have been reported. Moreover, the genetic 542 polymorphisms of both $C Y P 2 D 6$ and $C Y P 2 A 6$ could have a potential impact on the 543 magnitude of health benefits associated with the consumption of Tyr-containing food 544 amongst individuals and ethnicities. 
RFT has consulted for Apotex on unrelated issues. The other authors declare that

548 they have no conflict of interest.

549

550

551

552

553

554

555

556

557

558

559

560

561

562

563

\section{Acknowledgements}

The authors gratefully acknowledge the assistance of Professor J. Casabó (Universitat Autònoma de Barcelona) in performing data mining, multiple regression analysis, and 3D graphics.

This work was supported by grants from Instituto de Salud Carlos III FEDER, (PI14/00072), the CICYT-FEDER (AGL2009-13517-C03-01 and AGL2012-40144C03-01), grants from DIUE of the Generalitat de Catalunya (2014 SGR 680). CIBER de Fisiopatología de la Obesidad y Nutrición (CIBEROBN) is an initiative of the Instituto de Salud Carlos III, Madrid, Spain. JRM was supported by a FI-DGR2012 predoctoral fellowship from the Generalitat de Catalunya and CPM was supported by a Juan Rodés fellowship (ISCIII, JR, 15/00005). The authors acknowledge the support received from National Institutes of Health grant DA U01 020830, Canadian Institutes of Health Research grant MOP86471, the Endowed Chair in Addiction for the Department of Psychiatry University of Toronto (RFT), and funds from the Centre for Addiction and Mental Health (CAMH) and the CAMH Foundation (RFT). 
566 Al Koudsi, N., Hoffmann, E., Assadzadeh, A., \& Tyndale, R. (2010). Hepatic CYP2A6 levels and nicotine metabolism: impact of genetic, physiological, environmental, and epigenetic factors. European Journal of Clinical Pharmacology, 66 (3), 239-251.

Atkins, W. M. (2005). Non-Michaelis-Menten Kinetics in Cytochrome P450-Catalyzed Reactions. Annual Review of Pharmacology and Toxicology, 45 (1), 291-310.

Carrasco-Pancorbo, A., Cerretani, L., Bendini, A., Segura-Carretero, A., Del Carlo, M., Gallina-Toschi, T., Lercker, G., Compagnone, D., \& Fernández-Gutiérrez, A. (2005). Evaluation of the Antioxidant Capacity of Individual Phenolic Compounds in Virgin Olive Oil. Journal of Agricultural and Food Chemistry, 53 (23), 8918-8925.

Covas, M.-I., de la Torre, R., \& Fitó, M. (2015). Virgin olive oil: a key food for 578 cardiovascular risk protection. British Journal of Nutrition, 113 (SupplementS2), S19-S28. 307-310.

EFSA. Panel on Dietetic Products, Nutrition and Allergies (NDA); Scientific Opinion on the substantiation of health claims related to polyphenols in olive and protection of LDL particles from oxidative damage (ID 1333, 1638, 1639, 1696, 2865), maintenance of normal blood HDL-cholesterol concentrations (ID 1639), maintenance of normal blood pressure (ID 3781), "anti-inflammatory properties" (ID 1882), "contributes to the upper respiratory tract health" (ID 3468), "can help to maintain a normal function of gastrointestinal tract" (3779), and 
"contributes to body defences against external agents" (ID 3467) pursuant to

Article 13 (1) of Regulation (EC) No 1924/2006. EFSA J. 2011, 9, 2033,

online:

594 European Medicines Agency. (2012). URL:

http://www.ema.europa.eu/docs/en_GB/document_library/Scientific_guideline/2 012/07/WC500129606.pdf Accessed 17.11.2014.

Fernández-Mar, M. I., Mateos, R., García-Parrilla, M. C., Puertas, B., \& Cantos-Villar, 598 E. (2012). Bioactive compounds in wine: Resveratrol, hydroxytyrosol and melatonin: A review. Food Chemistry, 130 (4), 797-813.

600 Flores-Pérez, J., Flores-Pérez, C., Juárez-Olguín, H., Lares-Asseff, I., \& Sosa-Macías, 601 602

603 M. (2004). Determination of Dextromethorphan and Dextrorphan in Human Urine by High Performance Liquid Chromatography for Pharmacogenetic Reactions. Drug Metabolism and Disposition, 30 (4), 355-362.

610 Khymenets, O., Fitó, M., Touriño, S., Muñoz-Aguayo, D., Pujadas, M., Torres, J. L., 611 Joglar, J., Farré, M., Covas, M.-I., \& de la Torre, R. (2010). Antioxidant 612 Activities of Hydroxytyrosol Main Metabolites Do Not Contribute to Beneficial 613 Health Effects after Olive Oil Ingestion. Drug Metabolism and Disposition, 38 (9), 1417-1421. 
615 Khymenets, O., Joglar, J., Clapés, P., Parella, T., Covas, M.-I., \& de la Torre, R. (2006). Biocatalyzed Synthesis and Structural Characterization of Monoglucuronides of Hydroxytyrosol, Tyrosol, Homovanillic Alcohol, and 3-(4 ' Hydroxyphenyl)propanol. Advanced Synthesis \& Catalisis, 348 (15), 2155-2162.

619 Kotronoulas, A., Pizarro, N., Serra, A., Robledo, P., Joglar, J., Rubió, L., Hernaéz, Á., 620 Tormos, C., Motilva, M. J., Fitó, M., Covas, M.-I., Solà, R., Farré, M., Saez, G.,

Li, Y., Li, N. Y., \& Sellers, E. M. (1997). Comparison of CYP2A6 catalytic activity on 625 coumarin 7-hydroxylation in human and monkey liver microsomes. European Journal of Drug Metabolism and Pharmacokinetics, 22 (4), 295-304.

Messina, E. S., Tyndale, R. F., \& Sellers, E. M. (1997). A Major Role for CYP2A6 in Nicotine C-Oxidation by Human Liver Microsomes. Journal of Pharmacology and Experimental Therapeutics, 282 (3), 1608-1614.

Nagatsu, T., Levitt, M., \& Udenfriend, S. (1964). Tyrosine Hydroxylase. The Initial Step in Norepinephrine Biosynthesis. Journal of Biological Chemistry, 239, 2910-2917.

Pelkonen, O., Turpeinen, M., Hakkola, J., Honkakoski, P., Hukkanen, J., \& Raunio, H. (2008). Inhibition and induction of human cytochrome P450 enzymes: current status. Archives of Toxicology, 82 (10), 667-715.

Pérez-Mañá, C., Farre, M., Pujadas, M., Mustata, C., Menoyo, E., Pastor, A., Langohr, K., \& de la Torre, R. (2015). Ethanol induces hydroxytyrosol formation in humans. Pharmacological Research, 95-96, 27-33. 
Pérez-Mañá, C., Farré, M., Rodríguez-Morató, J., Papaseit, E., Pujadas, M., Fitó, M., Robledo, P., Covas, M. I., Cheynier, V., Meudec, E., Escudier, J. L., \& de la Torre, R. (2015). Moderate consumption of wine, through both its phenolic compounds and alcohol content, promotes hydroxytyrosol endogenous generation in humans. A randomized controlled trial. Molecular Nutrition \& Food Research, 59 (6), 1213-1216.

Piliguian, M., Zhu, A. Z. X., Zhou, Q., Benowitz, N. L., Ahluwalia, J. S., Cox, L. S., \& Tyndale, R. F. (2014). Novel CYP2A6 variants identified in African Americans are associated with slow nicotine metabolism in vitro and in vivo.

Piñeiro, Z., Cantos-Villar, E., Palma, M., \& Puertas, B. (2011). Direct Liquid Chromatography Method for the Simultaneous Quantification of Hydroxytyrosol and Tyrosol in Red Wines. Journal of Agricultural and Food Chemistry, 59 (21), 11683-11689.

Raunio, H., Rautio, A., Gullsten, H., \& Pelkonen, O. (2001). Polymorphisms of CYP2A6 and its practical consequences. British Journal of Clinical Pharmacology, 52 (4), 357-363.

Rautio, A., Kraul, H., Kojo, A., Salmela, E., \& Pelkonen, O. (1992). Interindividual 657 variability of coumarin 7-hydroxylation in healthy volunteers. Pharmacogenetics, 2 (5), 227-233.

Rodrigues, A. D. (2008). In Vitro Approaches for Studying the Inhibition of Drug660 Metabolizing Enzymes and Identifying the Drug-Metabolizing Enzymes 661 Responsible for the Metabolism of Drugs (Reaction Phenotyping) with Emphasis on Cytochrome P450. Drug-Drug Interactions, Chapter 7, 332-334.Rodríguez-Morató, J., Xicota, L., Fitó, M., Farré, M., Dierssen, M., \& de la Torre, R. (2015). 

Neurodegenerative Diseases. Molecules, 20 (3), 4655-4680.

Tank, A. W., \& Weiner, H. (1979). Ethanol-induced alteration of dopamine metabolism in rat liver. Biochemical Pharmacology, 28 (20), 3139-3147.

Tanner, J. A., Novalen, M., Jatlow, P., Huestis, M. A., Murphy, S. E., Kaprio, J., Kankaanpaa, A., Galanti, L., Stefan, C., George, T. P., Benowitz, N. L., Lerman, C., \& Tyndale, R. F. (2015). Nicotine metabolite ratio (3hydroxycotinine/cotinine) in plasma and urine by different analytical methods and laboratories: implications for clinical implementation. Cancer Epidemiology, Biomarkers \& Prevention, 24 (8), 1239-1246.

Teh, L. K., \& Bertilsson, L. (2012). Pharmacogenomics of CYP2D6: molecular genetics, interethnic differences and clinical importance. Drug Metabolism and Pharmacokinetics, 27 (1), 55-67.

Tyndale, R. F., Droll, K. P., \& Sellers, E. M. (1997). Genetically deficient CYP2D6 metabolism provides protection against oral opiate dependence. Pharmacogenetics, 7 (5), 375-379.

680 Tyndale, R. F., Inaba, T., \& Kalow, W. (1989). Evidence in humans for variant 681 allozymes of the nondeficient sparteine/debrisoquine monooxygenase (P45OIID 682 1) in vitro. Drug Metabolism and Disposition, 17 (3), 334-340. and strategies using real-time and end point PCR platforms. Pharmacogenomics, $17(2), 147-162$.

Yokoi, T., \& Kamataki, T. (1998). Genetic polymorphism of drug metabolizing 687 enzymes: new mutations in CYP2D6 and CYP2A6 genes in Japanese. Pharmaceutical Research, 15 (4), 517-524. 
689 Zhang, W., Kilicarslan, T., Tyndale, R. F., \& Sellers, E. M. (2001). Evaluation of 690 methoxsalen, tranylcypromine, and tryptamine as specific and selective 691 CYP2A6 inhibitors in vitro. Drug Metabolism and Disposition, 29 (6), 897-902.

692

693 


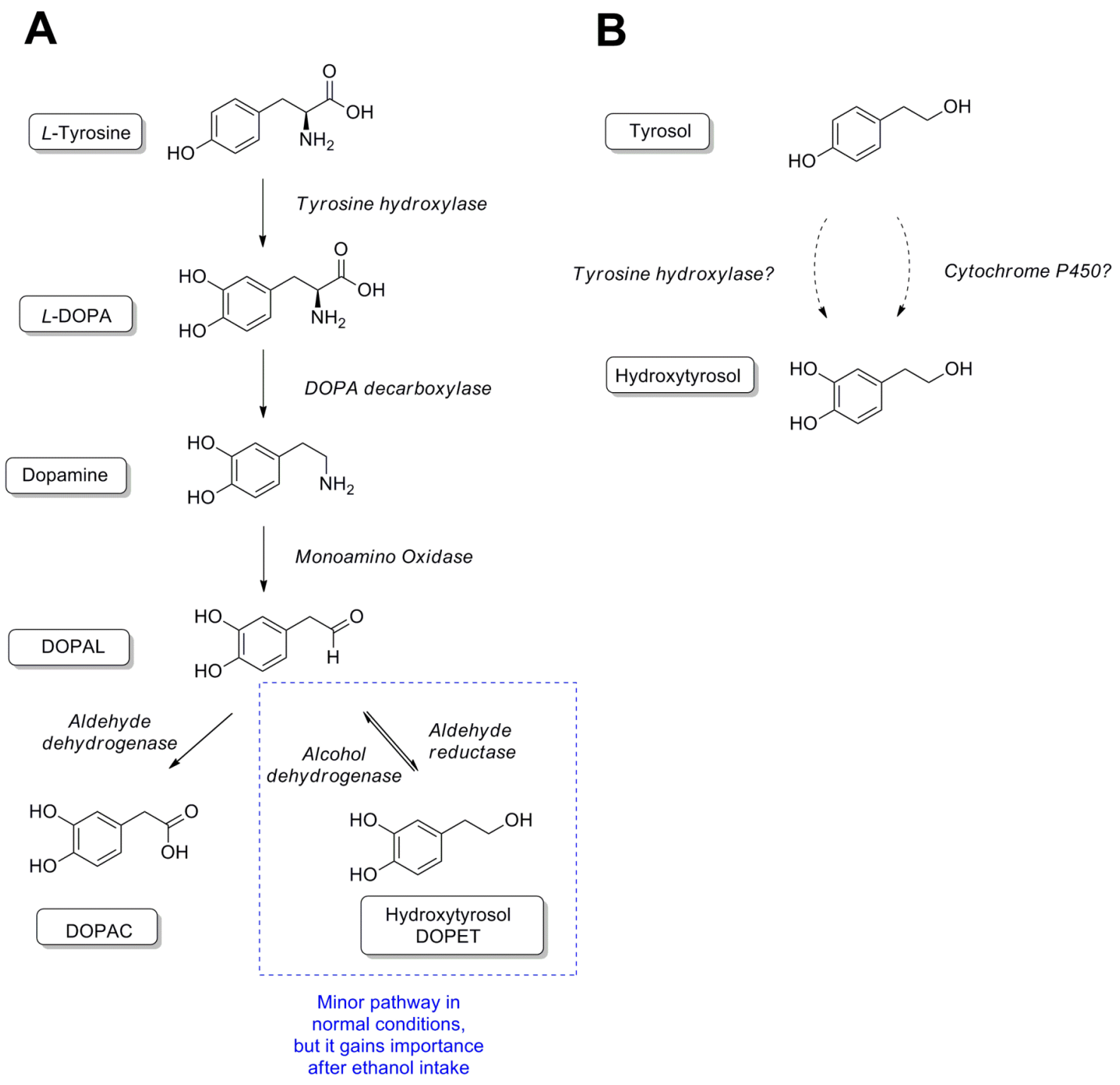

\section{Figure 1}

697 A, Dopamine biosynthesis and oxidative metabolism. Hydroxytyrosol is produced as a

698 metabolite of dopamine ( DOPET) and this formation is increased after ethanol intake in

699 humans. B, Schematic representation of the two hypotheses considered: (i) tyrosine

700 hydroxylase converts tyrosol into hydroxytyrosol as it converts tyrosine to $L$-DOPA; (ii)

701 cytochrome $\mathrm{P} 450$ is responsible for the hydroxylation. 

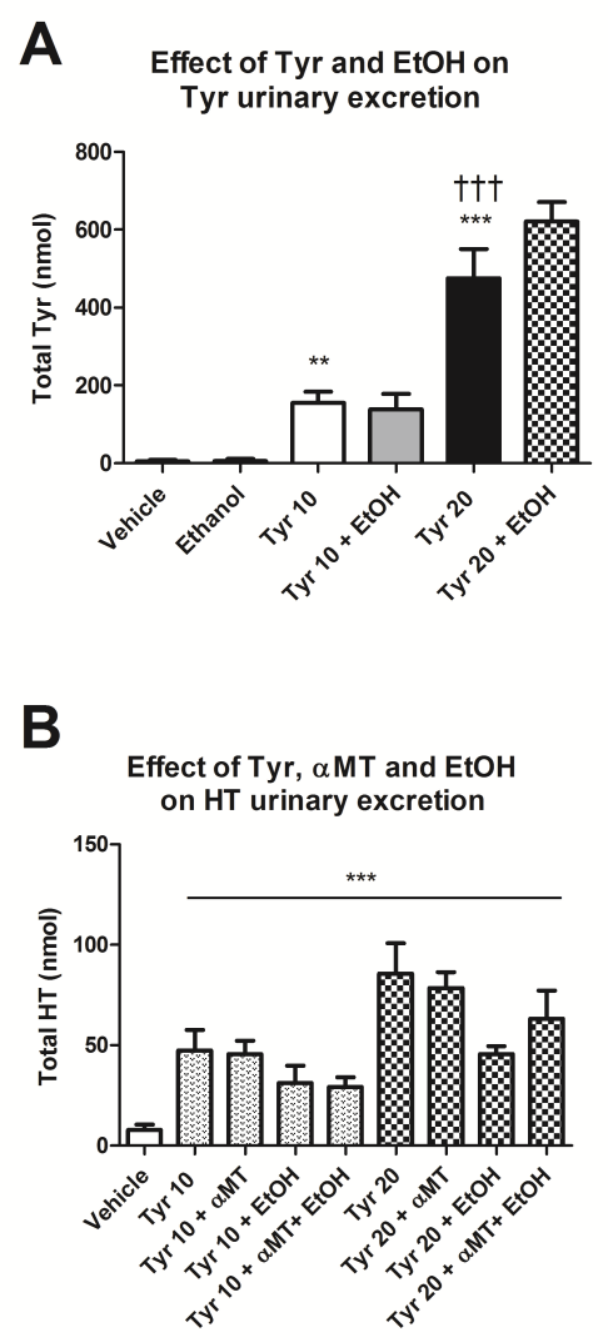

703

\section{Figure 2}

705 Total tyrosol (Tyr) and hydroxytyrosol (HT) urinary excretion (0-4 h) in rats. A, Tyr

706 urinary recoveries following Tyr administration with and without ethanol $(0.5 \mathrm{~g} / \mathrm{kg}) ; \mathbf{B}$,

707 HT urinary excretion following the administration of Tyr (10 and $20 \mathrm{mg} / \mathrm{kg}$ ), $\alpha$-methyl-

$708 \quad L$-tyrosine $(\alpha \mathrm{MT} ; 50 \mathrm{mg} / \mathrm{kg})$ and ethanol $(0.5 \mathrm{~g} / \mathrm{kg})$.

709 Data expressed as mean \pm SEM.

$710 * * P<0.005$, versus vehicle; $* * * P<0.001$, versus vehicle; ${ }^{\dagger \dagger} P<0.05$, versus Tyr 10

$711 \mathrm{mg} / \mathrm{kg}$

$712 \alpha \mathrm{MT}$ : $\alpha$-methyl-L-tyrosine; EtOH: Ethanol; Tyr 10: Tyrosol $10 \mathrm{mg} / \mathrm{kg}$; Tyr 20: Tyrosol

$71320 \mathrm{mg} / \mathrm{kg}$ 
A

HT formation in HLM

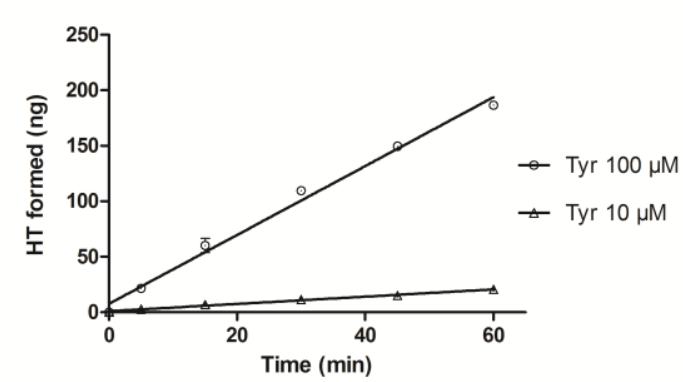

C

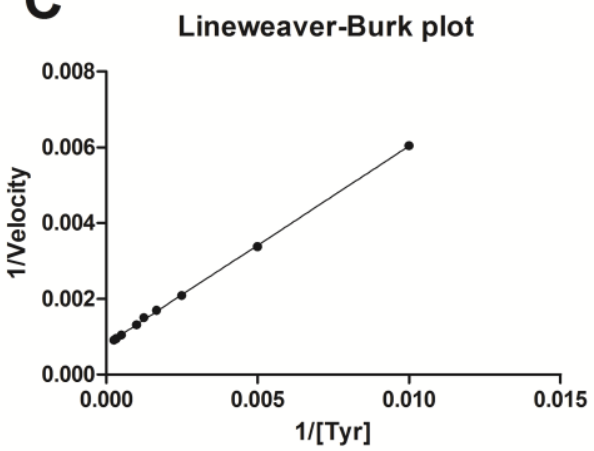

B Michaelis-Menten plot

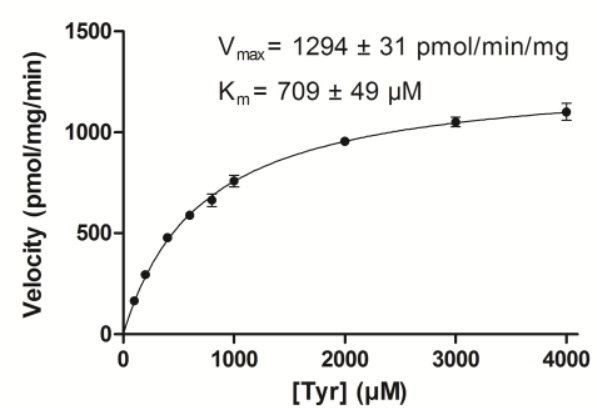

D Eadie-Hofstee plot

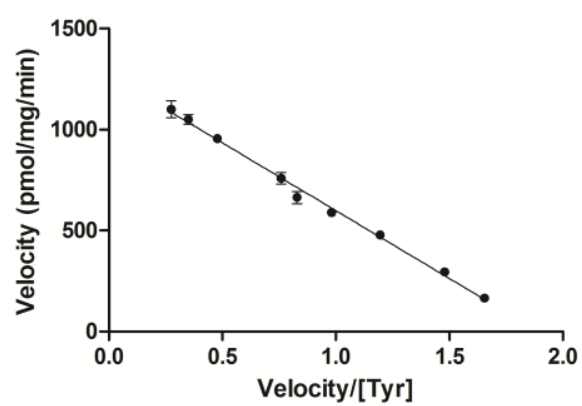

715

716 Figure 3

717 Hydroxytyrosol (HT) formation from tyrosol in human liver microsomes (HLM). A,

718 Time- and dose-dependent formation; B, Michaelis-Menten plot; C, Lineweaver-Burk

719 plot; D, Eadie-Hofstee plot.

720 


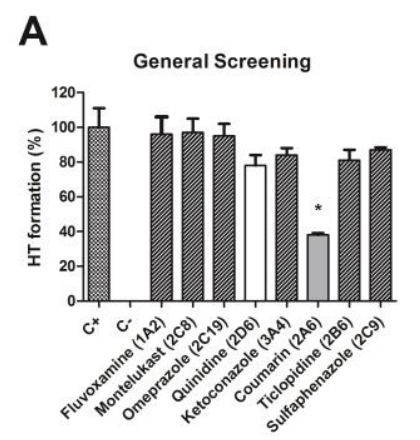

B

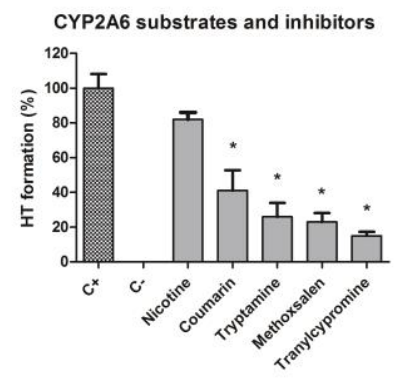

C

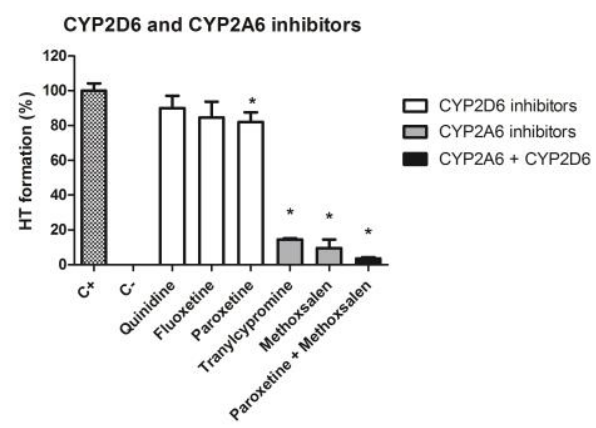

722 Figure 4

723 Inhibitory effect of cytochrome P450 (CYP) isoenzyme specific inhibitors on the

724 formation of hydroxytyrosol (HT) from tyrosol; A, Primary screening including

725 selective inhibitors of 8 different isoenzymes; B, Confirmatory incubations using

726 nicotine as a CYP2A6 substrate and selective CYP2A6 inhibitors (tryptamine,

727 methoxsalen and tranylcypromine); C, Additional experiments to evaluate the potential

728 effect of different inhibitors of CYP2A6 and CYP2D6. In this case, microsomes were

729 pre-incubated with inhibitors for $30 \mathrm{~min}$.

730 Values are given as mean \pm standard deviation of two independent experiments.

$731 * P<0.05$. 
A

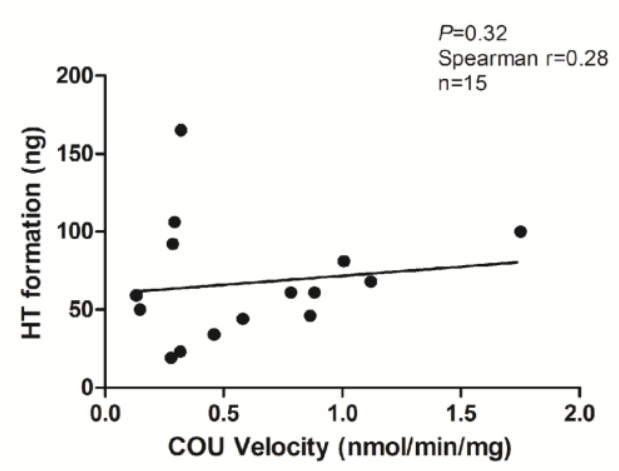

C

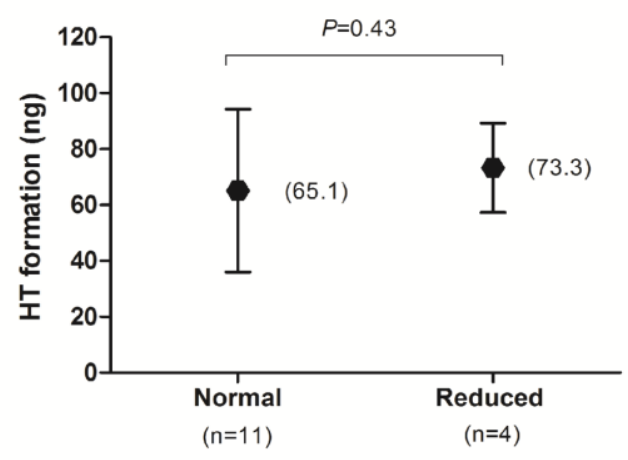

CYP2A6 Genotype Group

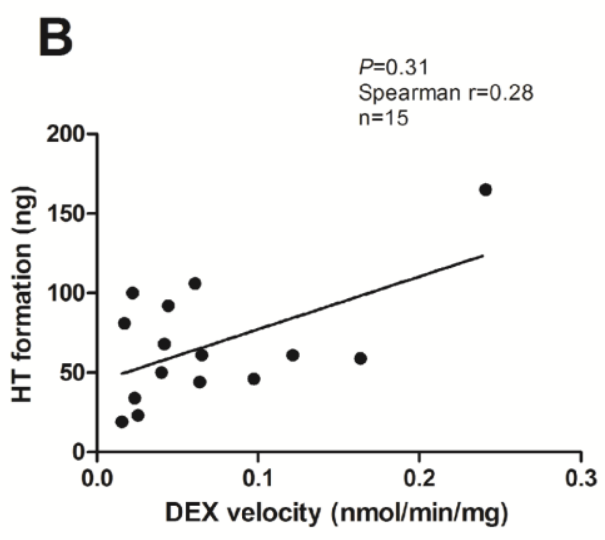

D

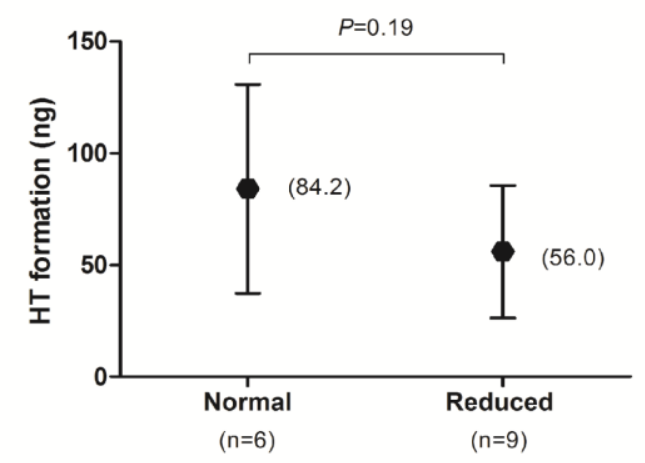

CYP2D6 Genotype Group

E

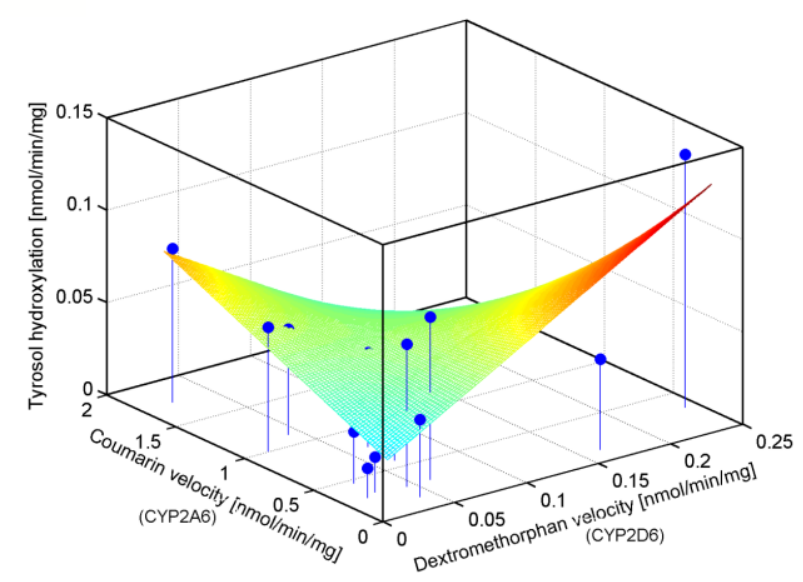




\section{Figure 5}

735 A. Correlation between hydroxytyrosol (HT) formation and CYP2A6 activity

736 (determined using the metabolism velocity of coumarin to 7-hydroxycoumarin).

737 B. Correlation between HT formation and CYP2D6 activity (determined using the

738 metabolism velocity of dextromethorphan to dextrorphan). $P$ and $\mathrm{r}$ values are based on

739 Spearman correlation test.

740 C. Association between CYP2A6 genotype and HT formation (ng). Normal

741 metabolizers are those with $* 1 / * 1, * 1 / * 1 X 2$, and $* 14 / * 14 C Y P 2 A 6$ genotypes. Reduced

742 metabolizers are those with $C Y P 2 A 6 * 1 / * 2$ and $* 1 / * 12$ genotypes.

743 D. Association between CYP2D6 genotype and HT formation (ng). Normal

744 metabolizers are those with $* 1 / * 1$ CYP2D6 genotype. Reduced metabolizers are those

745 with $C Y P 2 D 6 * 1 / * 3$, *1/*4, and $* 10 / * 10$ genotypes. $P$ values are based on Mann-

746 Whitney tests.

747 E. Multivariate correlation analysis of sample-to-sample variation $(\mathrm{n}=15)$ in tyrosol-3-

748 hydroxylation with CYP2D6 and CYPA6 activities. 